\title{
Diagnostic Value of Autoantibodies against Ezrin in Esophageal Squamous Cell Carcinoma
}

\author{
Lan Li, ${ }^{1}$ Ming Liu, ${ }^{1}$ Jian-Bang Lin, ${ }^{2}$ Xin-Bin Hong, ${ }^{1}$ Wen-Xia Chen, ${ }^{1}$ Hong Guo, ${ }^{3}$ \\ Li-Yan Xu, ${ }^{4}$ Yi-Wei Xu, ${ }^{5,6}$ En-Min Li, ${ }^{6,7}$ and Yu-Hui Peng ${ }^{5,6}$ \\ ${ }^{1}$ Shantou University Medical College, Shantou 515041, China \\ ${ }^{2}$ Department of Radiology, Cancer Hospital, Shantou University Medical College, Shantou 515041, China \\ ${ }^{3}$ Department of Radiation Oncology, Cancer Hospital, Shantou University Medical College, Shantou 515041, China \\ ${ }^{4}$ Institute of Oncologic Pathology, Shantou University Medical College, Shantou 515041, China \\ ${ }^{5}$ Department of Clinical Laboratory Medicine, Cancer Hospital, Shantou University Medical College, Shantou 515041, China \\ ${ }^{6}$ The Key Laboratory of Molecular Biology for High Cancer Incidence Coastal Chaoshan Area, Guangdong Higher Education Institutes, \\ Shantou University Medical College, Shantou 515041, China \\ ${ }^{7}$ Department of Biochemistry and Molecular Biology, Shantou University Medical College, Shantou 515041, China
}

Correspondence should be addressed to Yi-Wei Xu; yiwei512@126.com, En-Min Li; nmli@stu.edu.cn, and Yu-Hui Peng; pengyuhui666@163.com

Received 25 September 2016; Revised 10 January 2017; Accepted 24 January 2017; Published 16 February 2017

Academic Editor: Michele Malaguarnera

Copyright (C) 2017 Lan Li et al. This is an open access article distributed under the Creative Commons Attribution License, which permits unrestricted use, distribution, and reproduction in any medium, provided the original work is properly cited.

Esophageal squamous cell carcinoma (ESCC), one of the most common malignancies worldwide, is a highly aggressive and homogeneous entity occurring in esophageal squamous epithelium, and a reliable noninvasive test for early detection is needed. A recent study showed that serum autoantibodies against Ezrin could be detected in patients with pancreatic cancer. Here, we assessed whether autoantibodies against Ezrin could have diagnostic relevance for early ESCC. We analyzed autoantibodies against Ezrin in sera of 98 normal controls and 149 patients with ESCC. Ezrin autoantibodies levels were evaluated by enzyme-linked immunosorbent assay (ELISA). Results showed that higher levels of autoantibodies against Ezrin were observed in serum samples from patients with ESCC than in serum from normal controls $(P<0.0001)$. Based on a cutoff value of 0.319 , the sensitivity and specificity of autoantibodies against Ezrin for diagnosis of ESCC were $27.5 \%$ and $95.9 \%$, respectively. Compared with normal controls, the positive rate of autoantibodies against Ezrin was significantly elevated in patients with early-stage ESCC $(P<0.0001)$. Moreover, there was no significant difference of positivity of autoantibodies against Ezrin in ESCC patients categorized according to age, gender, tumor size, tumor invasion depth, tumor site, histological grade, lymph node status, or tumor stage. Our study indicates that the presence of autoantibodies against Ezrin is significantly associated with ESCC.

\section{Introduction}

Esophageal cancer is the 8th most common malignancy and the 6th leading cause of cancer-related mortality in the world [1]. Esophageal squamous cell carcinoma (ESCC) is the major subtype of esophageal cancer in China, which is one of the areas with the highest morbidity of esophageal cancer [2]. Incidences of esophageal squamous cell carcinoma (ESCC) have been reported to reach up to 100 cases per 100,000 annually in an area referred to as the "Asian esophageal cancer belt" (from northeast China to the Middle East) [2]. In spite of many advances in treatment, the 5-year survival rates for all patients diagnosed with esophageal cancer range from $15 \%$ to $20 \%$ [3]. This outcome is to some extent due to the lack of a screening approach for timely diagnosis. Indeed, ESCC patients often present at an advanced stage at the time of diagnosis when the tumor is no longer amenable to surgical resection $[3,4]$. Thus, a noninvasive screening procedure aiding in early ESCC diagnosis is urgently needed and would contribute to timely treatment in ESCC. 
In recent years, many studies have demonstrated that the antigenic changes of proteins in malignant cell, called tumor-associated antigens (TAAs), can be recognized by the immune system and further induce autoantibodies [5]. Importantly, autoantibodies seem to present at symptomatic stage of cancer, indicating the evaluation of autoantibodies viable for early cancer detection $[5,6]$.

Ezrin, a component of cell-surface structures pertaining to the member of ERM (Ezrin-Radixin-Moesin) family, acts as a linker between a number of growth factor receptors/adhesion molecules and the actin cytoskeleton [7]. Ezrin participates in cell-cell interactions and in cell adhesion to the extracellular matrix, $[8,9]$. There is also evidence that Ezrin is involved in signal transduction through Rho GTPase and receptor tyrosine-kinase signaling and interacted with cellular apoptotic machinery $[10,11]$. Ezrin is regarded as one of the promising key components in tumor metastasis, since it plays a role in interaction between the cell and its microenvironment, which facilitates intracellular signal transduction [12]. Ezrin is overexpressed in several kinds of cancers, which is associated with adverse outcomes [13-17]. Our previous study suggested that Ezrin might be a prognostic biomarker for ESCC [16]. Recently, Capello et al. identified autoantibodies to Ezrin as early diagnostic biomarker in pancreatic cancer [18]. Since evidences show that protein overexpression could induce humoral responses in cancer patients [19] and Ezrin proteins were found to be upregulated in ESCC (including early-stage ESCC) in comparison to adjacent normal tissue in our previous study [17], it is plausible that Ezrin might induce autoantibody production in ESCC patients.

To clarify the ability of autoantibodies against Ezrin in serum in ESCC diagnosis, we conducted enzyme-linked immunosorbent assay (ELISA) to investigate levels of autoantibodies against Ezrin in 149 ESCC patients and 98 normal controls and did western blot to confirm the results. We then explored the probable relevance between Ezrin autoantibodies and patients' clinical features.

\section{Methods}

2.1. Study Population. Patients with ESCC were recruited from the Cancer Hospital, Shantou University Medical College, Guangdong, China, from December 2013 to February 2015. The normal controls were from healthy individuals for physical examination at the Cancer Hospital, who had no previous and present evidence of malignant diseases based on imaging technology and had negative CEA and AFP results. The age and the sex in the patient group and the control group were matched as much as possible. Approval for the study from institutional review board of the Cancer Hospital of Shantou University Medical College and written informed consent from all patients and controls were obtained.

ESCC was defined by means of spiral computed tomography and gastroscopy and was histopathologically confirmed. Tumor stage was defined according to the seventh edition of the American Joint Committee on Cancer (AJCC) Cancer Staging Manual [20]. We classified tumors with AJCC stage $0+$ I as early-stage ESCC and AJCC stage II + III + IV as advanced ESCC.
Blood specimens from normal controls and newly diagnosed ESCC patients, collected at the time of diagnosis before any treatment, were centrifuged at $1250 \mathrm{~g}$ for $5 \mathrm{~min}$, and the serum samples were stored at $-80^{\circ} \mathrm{C}$ until use. Sera of patients with ESCC and normal controls were stored in a serum bank for research.

\subsection{Expression and Purification of Recombinant Ezrin Protein.} The coding sequence region for Ezrin (NM_003379) was subcloned into the pDEST17 expression vector (Invitrogen, Waltham, MA). The recombinant proteins, as described in our previous study, were expressed, purified, and analyzed $[21,22]$.

2.3. Autoantibody Detection. Two investigators (Lan Li and Ming Liu) who were blinded to clinical data of the patients and normal controls performed ELISA to measure serum autoantibodies as previously reported [21, 22]. In brief, purified Ezrin protein was diluted to $0.1 \mu \mathrm{g} / \mathrm{mL}$, and $100 \mu \mathrm{L}$ diluent was pipetted into each well to coat polystyrene plates. Self-made quality control samples and serum samples (at the dilution of $1: 110$ ) were incubated at $37^{\circ} \mathrm{C}$ for $1 \mathrm{~h}$, as well as appropriate polyclonal anti-rabbit antibodies (Cell Signaling Technology, Cat. No. 3145) specific for capture proteins. Secondary antibodies (HRP-conjugated goat anti-human/antimouse IgG) were added at the dilution of $1: 10000$. At color formation step, 3,3',5,5'-tetramethylbenzidine $(50 \mu \mathrm{L})$ and hydrogen peroxide $(50 \mu \mathrm{L})$ were add to each well. The cutoff value of positivity was defined as an $\mathrm{OD}_{450 \mathrm{~nm} / 630 \mathrm{~nm}}$ value greater than the mean plus 2 standard deviation (s.d.) from the 98 normal controls.

Quality control for monitoring of the ELISA assay was conducted according to our previous study [21,22].

2.4. Western Blot. Purified Ezrin protein was processed by electrophoretic separation on $10 \%$ SDS-PAGE and then transferred onto a polyvinylidene difluoride (PVDF) membranes by the iBlot $^{\circledR} 2$ Dry Blotting System (Thermo Fisher Scientific). The PVDF membranes were incubated in blocking buffer (TBS containing 0.05\% Tween-20 (TBST) and 5\% nonfat dry milk) at room temperature for $1 \mathrm{~h}$ and were then incubated overnight at $4^{\circ} \mathrm{C}$ with a $1: 100$ dilution of serum and 1:2000 dilution of polyclonal anti-Ezrin antibody. We used HRP-conjugated goat anti-human/anti-mouse IgG as secondary antibodies at the dilution of 1:5000 which was recommended by the manufacturer. Finally, we detected immunoreactive bands by using the ECL kit (Thermo Fisher Scientific), which were photographed by FluorChem 8900 (Alpha Innotech, USA).

2.5. Statistical Analysis. We conducted all analyses with the use of the GraphPad Prism 5 software, Microsoft Excel, and SPSS (version 17.0). The nonparametric Mann-Whitney $U$ test was used to compare the Ezrin autoantibody levels between sera of normal controls and ESCC. Chi-squared tests were used for the comparison of positive rates between cancer patients and control group and to evaluate relationships of test positivity with clinicopathological features. In all tests, we considered $P$ values of $<0.05$ (two sided) to be significant. 
TABLE 1: Participant details and clinicopathological features.

\begin{tabular}{|c|c|c|}
\hline Group & ESCC & Normal \\
\hline Number & 149 & 98 \\
\hline Male, $n(\%)$ & $107(72 \%)$ & $70(71 \%)$ \\
\hline Female, $n(\%)$ & $42(28 \%)$ & $28(29 \%)$ \\
\hline Mean age \pm s.d. (years) & $58 \pm 9$ & $55 \pm 10$ \\
\hline Age range (years) & $41-88$ & $34-87$ \\
\hline \multicolumn{3}{|l|}{ Tumor invasion depth } \\
\hline Tis & 4 & \\
\hline $\mathrm{T} 1+\mathrm{T} 2$ & 32 & \\
\hline $\mathrm{T} 3+\mathrm{T} 4$ & 113 & \\
\hline \multicolumn{3}{|l|}{ Lymph node metastasis } \\
\hline Positive & 72 & \\
\hline Negative & 77 & \\
\hline \multicolumn{3}{|l|}{ Histological grade } \\
\hline High & 40 & \\
\hline Medium & 93 & \\
\hline Low & 16 & \\
\hline \multicolumn{3}{|l|}{ TNM stage } \\
\hline 0 & 4 & \\
\hline I & 14 & \\
\hline II & 57 & \\
\hline III & 44 & \\
\hline III & 3 & \\
\hline \multicolumn{3}{|l|}{ Tumor size } \\
\hline$<5 \mathrm{~cm}$ & 89 & \\
\hline$\geq 5 \mathrm{~cm}$ & 60 & \\
\hline \multicolumn{3}{|l|}{ Tumor site } \\
\hline Upper thorax & 17 & \\
\hline Middle thorax & 111 & \\
\hline Lower thorax & 21 & \\
\hline
\end{tabular}

ESCC: esophageal squamous cell carcinoma.

\section{Results}

3.1. Serum Level and Frequency of Autoantibodies against Ezrin in ESCC Patients. We in total obtained 247 serum samples for this study, 149 in patient group and 98 in control group. The demographics and characteristics of ESCC patients and normal controls are summarized in Table 1.

The OD values of autoantibodies against Ezrin for ESCC patients and normal controls ranged from 0.048 to 0.863 and from 0.012 to 0.389 , respectively. The mean $\mathrm{OD} \pm$ s.d. of serum autoantibodies against Ezrin was $0.258 \pm 0.153$ in the 149 ESCC patients and $0.177 \pm 0.071$ in the 98 normal controls. Figure 1 clearly shows that serum autoantibodies levels were higher in the ESCC patients than in normal controls $(P<$ 0.0001). Box plots and Scatter plots of OD values of Ezrin autoantibodies in sera of ESCC patients by AJCC stage were also shown in Figure 1. As shown in Table 2, when we used a cutoff value of 0.319 , the positive rate of autoantibody against Ezrin was 27.5\% in ESCC, which was significantly higher than that in normal controls $(P<0.0001)$. We next validated the ELISA results using western blot analysis. Compared to
TABLE 2: Positive rates of autoantibodies against Ezrin for ESCC.

\begin{tabular}{lccc}
\hline Group & $N$ & Positive (\%) & $P$ value \\
\hline ESCC & 149 & $41(27.5 \%)$ & $<0.0001$ \\
Early-stage ESCC (0 + I) & 18 & $5(27.8 \%)$ & $<0.0001$ \\
Advanced ESCC (II + III + IV) & 131 & $36(27.5 \%)$ & $<0.0001$ \\
Normal controls & 98 & $4(4.1 \%)$ & \\
\hline
\end{tabular}

ESCC: esophageal squamous cell carcinoma. $P$ value is relative to normal controls. Statistical significance was determined using the $\chi^{2}$ test.

normal serum, representative serum from ESCC patients also showed strong reactivity in western blotting (Figure 2), which were detected with positive results to Ezrin autoantibodies by ELISA method. Detection of this autoantibody assay provided a sensitivity of $27.5 \%$, with a robust specificity of 95.9\% (Table 3).

3.2. Diagnostic Value of Autoantibodies against Ezrin for Early ESCC and Advanced ESCC. In this study, there are 18 patients with early-stage ESCC (AJCC stage $0+$ I) and 131 with advanced ESCC (AJCC stage II + III + IV), respectively. Chi-squared test showed that patients with early-stage ESCC had a higher positive rate for this autoantibody test than normal controls $(P<0.0001$, Table 2$)$. Similar result was also observed in advanced ESCC patients, when compared with normal controls (Table 2). The ability of autoantibodies against Ezrin to diagnose early ESCC or advanced ESCC was also evaluated (Table 3). Moreover, 1 of 4 ESCC patients (25\%) who were defined to have stage 0 disease showed a positive result of autoantibody detection.

3.3. Effect of Clinicopathological Features on Biomarker Assay. We further assessed the differences of the Ezrin autoantibody positivity with clinical variables in ESCC patients, and we found that there were no significant correlations in assay positivity with patient age, gender, tumor size, tumor invasion depth, tumor site, histological grade, lymph node status, TNM stage or early-stage, and advanced stage groups (all $P>0.05$, Table 4$)$.

\section{Discussion}

ESCC lacks particular symptoms at early stage and effective noninvasive methods for screening, leading to the present situation that the detection of early ESCC is hampered [3]. Currently, the use of endoscopic screening and treatment has contributed to reduction in ESCC-associated mortality, but the early detection based on a noninvasive procedure (e.g., serum biomarker) is still imminently needed to improve the outcomes of these patients. In clinical practice, squamous cell carcinoma antigen (SCCA), carcinoembryonic antigen (CEA), and CYFRA 21-1 are used as serum tumor markers for ESCC, but low sensitivity and low specificity limit their application in the early diagnosis [23-25]. In this study, we report an ELISA assay to identify Ezrin proteins that induce a humoral response in ESCC, showing potential utility of autoantibodies against Ezrin in ESCC diagnosis. 
TABLE 3: Diagnostic results for autoantibodies against Ezrin in ESCC.

\begin{tabular}{|c|c|c|c|c|c|c|}
\hline & Sensitivity & Specificity & PPV & NPV & PLR & NLR \\
\hline ESCC versus NC & $27.5 \%$ & $95.9 \%$ & $91.1 \%$ & $46.5 \%$ & 6.74 & 0.76 \\
\hline Early-stage ESCC versus NC & $27.8 \%$ & $95.9 \%$ & $55.4 \%$ & $87.9 \%$ & 6.78 & 0.75 \\
\hline Advanced ESCC versus NC & $27.5 \%$ & $95.9 \%$ & $90.0 \%$ & $49.7 \%$ & 6.71 & 0.76 \\
\hline
\end{tabular}

ESCC: esophageal squamous cell carcinoma; NC: normal controls; NLR: negative likelihood ratio; NPV: negative predictive value; PLR: positive likelihood ratio; PPV: positive predictive value.

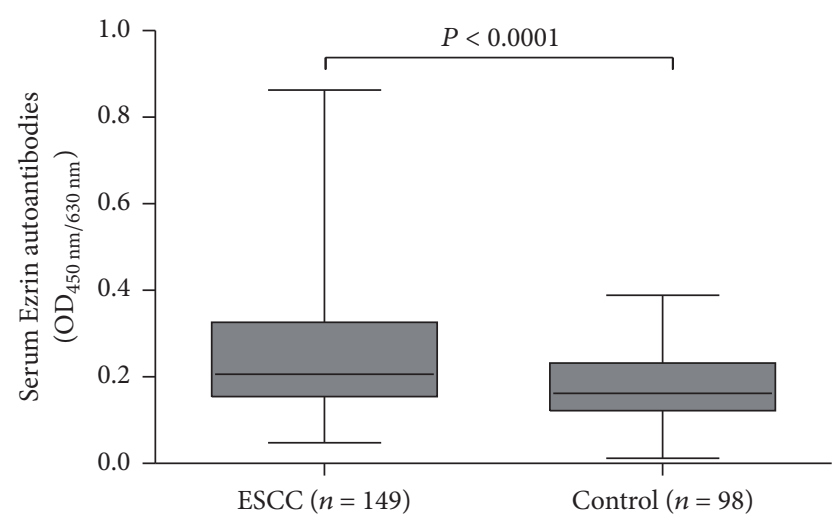

(a)

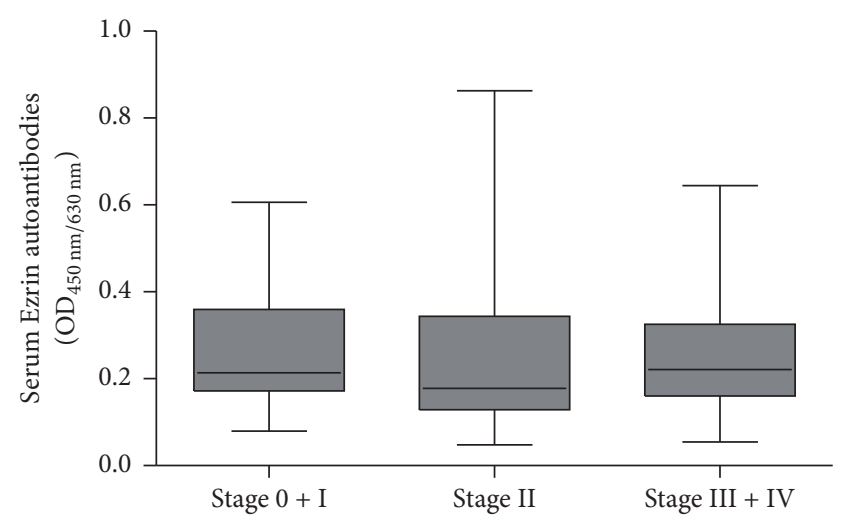

(c)

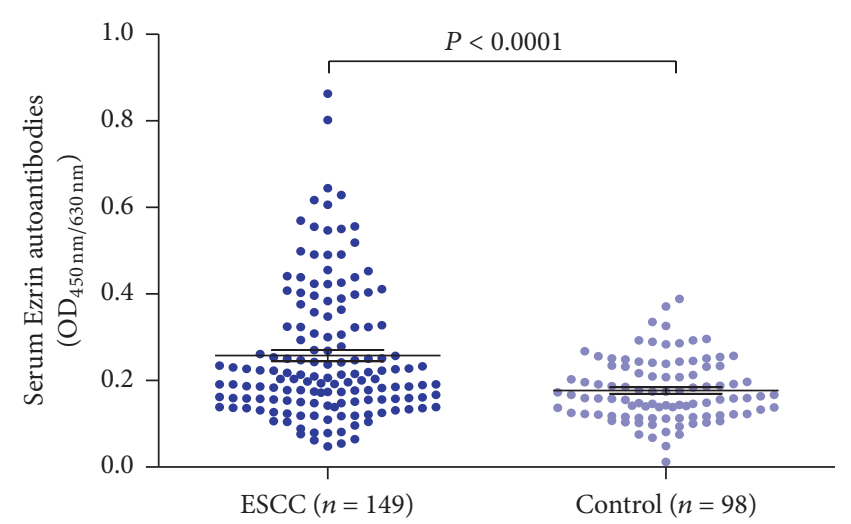

(b)

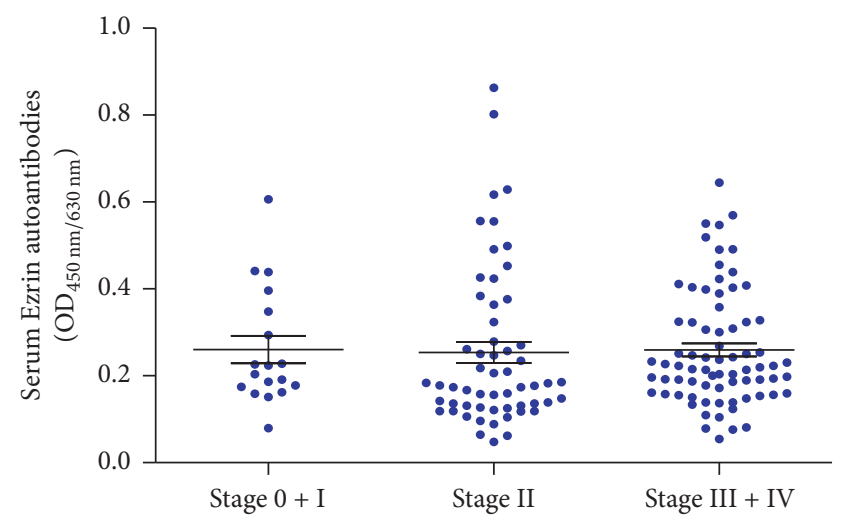

(d)

FIGURE 1: levels of serum Ezrin autoantibodies. (a) Median levels and interquartile ranges of serum Ezrin autoantibodies in ESCC patients and normal controls are illustrated by box plot and the whiskers show minimum and maximum value. (b) Scatter plots of OD values of Ezrin autoantibodies from sera of ESCC patients and normal controls. Black horizontal lines are means, and error bars are SEs. (c) Median levels and interquartile ranges of serum Ezrin autoantibodies in ESCC patients by AJCC stage are illustrated by box plot and the whiskers show minimum and maximum value. (d) Scatter plots of OD values of Ezrin autoantibodies from sera of ESCC patients by AJCC stage. Black horizontal lines are means, and error bars are SEs.

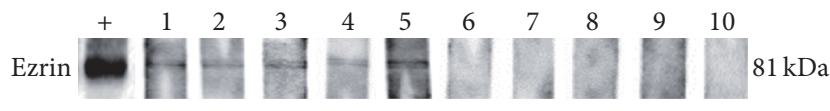

FIGURE 2: Western blotting analysis with representative sera recognizing Ezrin recombinant protein. The polyclonal anti-Ezrin antibody was used as positive control; lanes 1-5, five representative ESCC sera (with AJCC stage I, stage II, stage II, stage III, and stage III, resp.) with positive results in ELISA test have strong reactivity with Ezrin recombinant protein in western blotting analysis; lanes 6-10 are five representative normal control sera with negative reactivity.
In recent years, autoantibodies to TAAs have drawn increasing scientific interest owing to their promising value of clinical application in terms of the early detection of cancer $[5,6,21,22,26-30]$. As a matter of fact, biomarkers for early diagnosis of cancer require high specificity and sensitivity, but the detection of a single autoantibody appears to lack enough diagnostic power $[5,26]$. An increasing number of studies have suggested that a panel of several autoantibodies, to be measured simultaneously, may overcome this issue. For example, the EarlyCDT-Lung test has shown that continuous 
TABLE 4: Relationship between positive rate of the autoantibody and clinicopathologic features in ESCC patients.

\begin{tabular}{|c|c|c|c|}
\hline Group & $n$ & Positive (\%) & $P$ value \\
\hline \multicolumn{4}{|l|}{ Patient age } \\
\hline$\leq 55$ & 57 & $11(19.3 \%)$ & \multirow[t]{2}{*}{0.077} \\
\hline$>55$ & 92 & $30(32.6 \%)$ & \\
\hline \multicolumn{4}{|l|}{ Patient gender } \\
\hline Female & 42 & $12(28.6 \%)$ & \multirow[t]{2}{*}{0.857} \\
\hline Male & 107 & $29(27.1 \%)$ & \\
\hline \multicolumn{4}{|c|}{ Tumor invasion Depth } \\
\hline $\mathrm{T} 1+\mathrm{T} 2$ & 32 & $10(31.3 \%)$ & \multirow[t]{2}{*}{0.599} \\
\hline $\mathrm{T} 3+\mathrm{T} 4$ & 113 & $30(26.5 \%)$ & \\
\hline \multicolumn{4}{|c|}{ Lymph node metastasis } \\
\hline Positive & 72 & $18(25.0 \%)$ & \multirow[t]{2}{*}{0.506} \\
\hline Negative & 77 & $23(29.9 \%)$ & \\
\hline \multicolumn{4}{|l|}{ Histological grade } \\
\hline High & 40 & $8(20.0 \%)$ & \multirow[t]{3}{*}{0.274} \\
\hline Medium & 93 & $30(32.3 \%)$ & \\
\hline Low & 15 & $3(20.0 \%)$ & \\
\hline \multicolumn{4}{|l|}{ TNM stage } \\
\hline I & 14 & $4(28.6 \%)$ & \multirow[t]{4}{*}{0.234} \\
\hline II & 57 & $15(26.3 \%)$ & \\
\hline III & 44 & $20(45.5 \%)$ & \\
\hline IV & 3 & $1(33.3 \%)$ & \\
\hline \multicolumn{4}{|l|}{ Tumor size } \\
\hline$<5 \mathrm{~cm}$ & 89 & $27(30.3 \%)$ & \multirow[t]{2}{*}{0.348} \\
\hline$\geq 5 \mathrm{~cm}$ & 60 & $14(23.3 \%)$ & \\
\hline \multicolumn{4}{|l|}{ Tumor Site } \\
\hline Upper thorax & 17 & $3(17.6 \%)$ & \multirow[t]{3}{*}{0.364} \\
\hline Middle thorax & 111 & $30(27.0 \%)$ & \\
\hline Lower thorax & 21 & $8(38.1 \%)$ & \\
\hline \multicolumn{4}{|c|}{ Early-stage versus advanced-stage } \\
\hline Early-stage & 18 & $5(27.8 \%)$ & \multirow[t]{2}{*}{0.979} \\
\hline Advanced-stage & 131 & $36(27.5 \%)$ & \\
\hline
\end{tabular}

Statistical significance was determined by means of Chi-squared test.

addition of antigen up to seven TAAs in total increased the sensitivity to $41 \%$ in patients with lung cancer, but almost did not decrease the specificity in control samples [30]. Our study reported similar findings, which demonstrated that using a panel of six TAAs (i.e., BMI-1, PRDX6, NY-ESO-1, p53, HSP70, and MMP-7) could distinguish patients with early ESCC from normal controls, with a sensitivity/specificity of $45 \% / 95 \%$ [21]. On the other hand, it should be emphasized that the clinical application of autoantibodies in early cancer diagnosis might rely on an optimized panel of TAAs selected with high specificity and sensitivity. We here measured autoantibodies against Ezrin in sera of patients with earlystage ESCC and normal controls resulting in $27.8 \%$ sensitivity with a robust specificity of $95.9 \%$. The robust specificity should make this autoantibody assay possible to be selected for combining an optimized autoantibody panel which is useful in early ESCC diagnosis. When we attempted to combine Ezrin autoantibodies with autoantibodies against p53 and NY-ESO-1 which were integral to our identified autoantibody panel in previous study [21], we found that this combination presented an enhanced sensitivity with the same specificity in early ESCC diagnosis compared with either test alone (data not shown). However, the size of patients with early disease in the present study was relatively small. Thus, we need to enlarge the number of early-stage ESCC to further assess the early diagnostic ability of Ezrin autoantibody in future study.

Expression of Ezrin may be upregulated in cancer, and most probably phosphorylation of Ezrin is upregulated, which is shown to play an important role in tumor cell differentiation and metastasis [31]. Recently, a recent pancreatic cancer study reported firstly by Capello et al. provided evidence that Ezrin autoantibodies exists early in the stage of carcinogenesis [18]. These studies support the immunogenicity of Ezrin that we observed in ESCC in the present study, even though the mechanism on how autoreactive immune responses against Ezrin are induced is not clear.

In conclusion, to date this study is the first to assess the diagnostic value of serum autoantibodies against Ezrin in ESCC, indicating that the presence of autoantibodies against Ezrin is significantly associated with ESCC and may be of clinical value for ESCC. Moreover, our results reveal that autoantibody against Ezrin combined with other specific autoantibodies in esophageal cancer may shed light on a promising way to develop a highly sensitive test for early diagnosis of ESCC in the future.

\section{Competing Interests}

The authors declare that they have no competing interests.

\section{Authors' Contributions}

Lan Li and Ming Liu contributed equally to this work.

\section{Acknowledgments}

This work was supported by the National Science Foundation of China (nos. 81172264, 81472613, and 81360331), the Shantou University Medical College Clinical Research Enhancement Initiative (201428), the Undergraduate Innovation Program of Guangdong (201510560027), the Innovative and Strong School Project of Guangdong (2015KQNCX044), the Natural Science Foundation of China-Guangdong Joint Fund (nos. U1301227 and U0932001), and the Department of Education, Guangdong Government under the Top-tier University Development Scheme for Research and Control of Infectious Diseases.

\section{References}

[1] J. Ferlay, I. Soerjomataram, R. Dikshit et al., "Cancer incidence and mortality worldwide: sources, methods and major patterns in GLOBOCAN 2012," International Journal of Cancer, vol. 136, no. 5, pp. E359-E386, 2015. 
[2] L. A. Torre, F. Bray, R. L. Siegel, J. Ferlay, J. Lortet-Tieulent, and A. Jemal, "Global cancer statistics, 2012," CA Cancer Journal for Clinicians, vol. 65, no. 2, pp. 87-108, 2015.

[3] S. Ohashi, S. Miyamoto, O. Kikuchi, T. Goto, Y. Amanuma, and M. Muto, "Recent advances from basic and clinical studies of esophageal squamous cell carcinoma," Gastroenterology, vol. 149, no. 7, pp. 1700-1715, 2015.

[4] W.-Q. Wei, Z.-F. Chen, Y.-T. He et al., "Long-term follow-up of a community assignment, one-time endoscopic screening study of esophageal cancer in China," Journal of Clinical Oncology, vol. 33, no. 17, pp. 1951-1957, 2015.

[5] H. T. Tan, J. Low, S. G. Lim, and M. C. M. Chung, "Serum autoantibodies as biomarkers for early cancer detection," FEBS Journal, vol. 276, no. 23, pp. 6880-6904, 2009.

[6] W. Liu, B. Peng, Y. Lu, W. Xu, W. Qian, and J.-Y. Zhang, "Autoantibodies to tumor-associated antigens as biomarkers in cancer immunodiagnosis," Autoimmunity Reviews, vol. 10, no. 6, pp. 331-335, 2011.

[7] R. G. Fehon, A. I. McClatchey, and A. Bretscher, "Organizing the cell cortex: the role of ERM proteins," Nature Reviews Molecular Cell Biology, vol. 11, no. 4, pp. 276-287, 2010.

[8] K. Krishnan, B. Bruce, S. Hewitt, D. Thomas, C. Khanna, and L. J. Helman, "Ezrin mediates growth and survival in Ewing's sarcoma through the AKT/mTOR, but not the MAPK, signaling pathway," Clinical and Experimental Metastasis, vol. 23, no. 3-4, pp. 227-236, 2006.

[9] D. Brambilla, S. Zamboni, C. Federici et al., "P-glycoprotein binds to ezrin at amino acid residues 149-242 in the FERM domain and plays a key role in the multidrug resistance of human osteosarcoma," International Journal of Cancer, vol. 130, no. 12, pp. 2824-2834, 2012.

[10] X. Wan, S. Y. Kim, L. M. Guenther et al., "Beta4 integrin promotes osteosarcoma metastasis and interacts with ezrin," Oncogene, vol. 28, no. 38, pp. 3401-3411, 2009.

[11] S. Monferran, N. Skuli, C. Delmas et al., " $\alpha \mathrm{v} \beta 3$ and $\alpha \mathrm{v} \beta 5$ integrins control glioma cell response to ionising radiation through ILK and RhoB," International Journal of Cancer, vol. 123, no. 2, pp. 357-364, 2008.

[12] H.-R. Park, R. L. Cabrini, E. S. Araujo, M. L. Paparella, D. Brandizzi, and Y.-K. Park, "Expression of ezrin and metastatic tumor antigen in osteosarcomas of the jaw," Tumori, vol. 95, no. 1, pp. 81-86, 2009.

[13] N. Torer, F. Kayaselcuk, T. Z. Nursal et al., "Adhesion molecules as prognostic markers in pancreatic adenocarcinoma," Journal of Surgical Oncology, vol. 96, no. 5, pp. 419-423, 2007.

[14] L. Ma and T. Jiang, "Clinical implications of Ezrin and CD44 co-expression in breast cancer," Oncology Reports, vol. 30, no. 4, pp. 1899-1905, 2013.

[15] Y. Meng, Z. Lu, S. Yu, Q. Zhang, Y. Ma, and J. Chen, "Ezrin promotes invasion and metastasis of pancreatic cancer cells," Journal of Translational Medicine, vol. 8, article 61, 2010.

[16] J.-J. Xie, L.-Y. Xu, Z.-Y. Wu et al., "Prognostic implication of ezrin expression in esophageal squamous cell carcinoma," Journal of Surgical Oncology, vol. 104, no. 5, pp. 538-543, 2011.

[17] J.-J. Xie, L.-Y. Xu, Y.-M. Xie et al., "Roles of ezrin in the growth and invasiveness of esophageal squamous carcinoma cells," International Journal of Cancer, vol. 124, no. 11, pp. 25492558, 2009.

[18] M. Capello, P. Cappello, F. C. Linty et al., "Autoantibodies to Ezrin are an early sign of pancreatic cancer in humans and in genetically engineered mouse models," Journal of Hematology and Oncology, vol. 6, no. 1, article no. 67, 2013.
[19] K. S. Anderson and J. LaBaer, "The sentinel within: exploiting the immune system for cancer biomarkers," Journal of Proteome Research, vol. 4, no. 4, pp. 1123-1133, 2005.

[20] T. W. Rice, E. H. Blackstone, and V. W. Rusch, "Editorial: 7th edition of the AJCC cancer staging manual: esophagus and esophagogastric junction," Annals of Surgical Oncology, vol. 17, no. 7, pp. 1721-1724, 2010.

[21] Y.-W. Xu, Y.-H. Peng, B. Chen et al., "Autoantibodies as potential biomarkers for the early detection of esophageal squamous cell carcinoma," American Journal of Gastroenterology, vol. 109, no. 1, pp. 36-45, 2014.

[22] Y.-H. Peng, Y.-W. Xu, S.-Q. Qiu et al., "Combination of autoantibodies against NY-ESO-1 and viral capsid antigen immunoglobulin A for improved detection of nasopharyngeal carcinoma," Oncology Letters, vol. 8, no. 3, pp. 1096-1102, 2014.

[23] K. Mealy, J. Feely, I. Reid, J. McSweeney, T. Walsh, and T. P. J. Hennessy, "Tumour marker detection in oesophageal carcinoma," European Journal of Surgical Oncology, vol. 22, no. 5, pp. 505-507, 1996.

[24] S.-I. Kosugi, T. Nishimaki, T. Kanda, S. Nakagawa, M. Ohashi, and K. Hatakeyama, "Clinical significance of serum carcinoembryonic antigen, carbohydrate antigen 19-9, and squamous cell carcinoma antigen levels in esophageal cancer patients," World Journal of Surgery, vol. 28, no. 7, pp. 680-685, 2004.

[25] H. Shimada, Y. Nabeya, S.-I. Okazumi et al., "Prognostic significance of CYFRA 21-1 in patients with esophageal squamous cell carcinoma," Journal of the American College of Surgeons, vol. 196, no. 4, pp. 573-578, 2003.

[26] S. Werner, H. Chen, S. Tao, and H. Brenner, "Systematic review: serum autoantibodies in the early detection of gastric cancer," International Journal of Cancer, vol. 136, no. 10, pp. 2243-2252, 2015.

[27] H. Zhang, J. Xia, K. Wang, and J. Zhang, "Serum autoantibodies in the early detection of esophageal cancer: a systematic review," Tumor Biology, vol. 36, no. 1, pp. 95-109, 2015.

[28] S. Lam, P. Boyle, G. F. Healey et al., "EarlyCDT-Lung: an immunobiomarker test as an aid to early detection of lung cancer," Cancer Prevention Research, vol. 4, no. 7, pp. 1126-1134, 2011.

[29] J. Qiu, G. Choi, L. Li et al., "Occurrence of autoantibodies to annexin I, 14-3-3 theta and LAMR1 in prediagnostic lung cancer sera," Journal of Clinical Oncology, vol. 26, no. 31, pp. 5060-5066, 2008.

[30] C. J. Chapman, G. F. Healey, A. Murray et al., "EarlyCDT ${ }^{\circledR}$-Lung test: improved clinical utility through additional autoantibody assays," Tumor Biology, vol. 33, no. 5, pp. 1319-1326, 2012.

[31] T. Jin, J. Jin, X. Li et al., "Prognostic implications of ezrin and phosphorylated ezrin expression in non-small cell lung cancer," BMC Cancer, vol. 14, no. 1, article no. 191, 2014. 


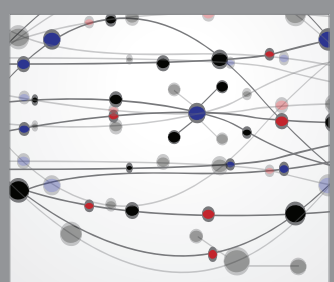

The Scientific World Journal


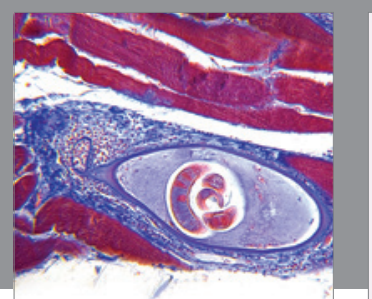

Gastroenterology Research and Practice
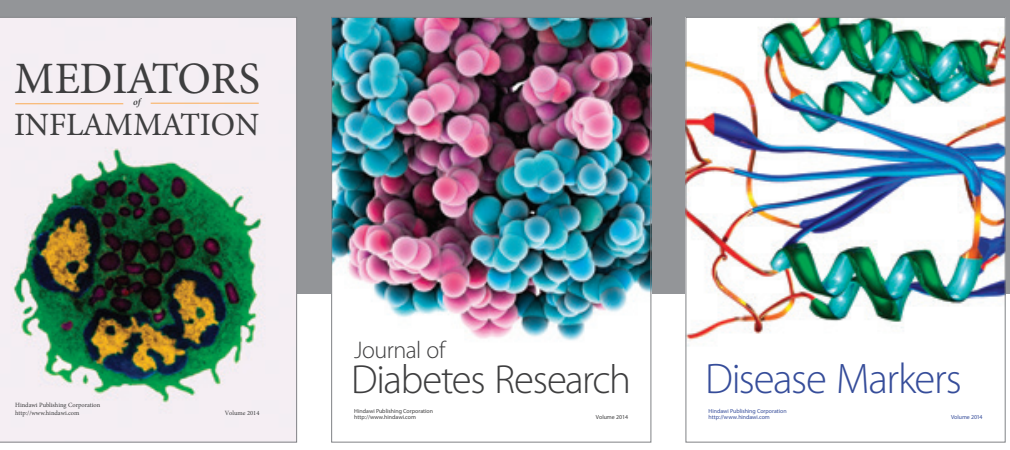

Disease Markers

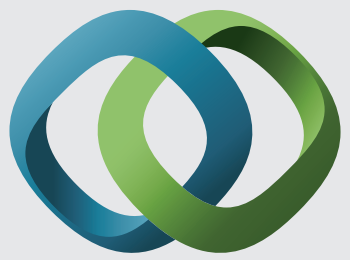

\section{Hindawi}

Submit your manuscripts at

https://www.hindawi.com
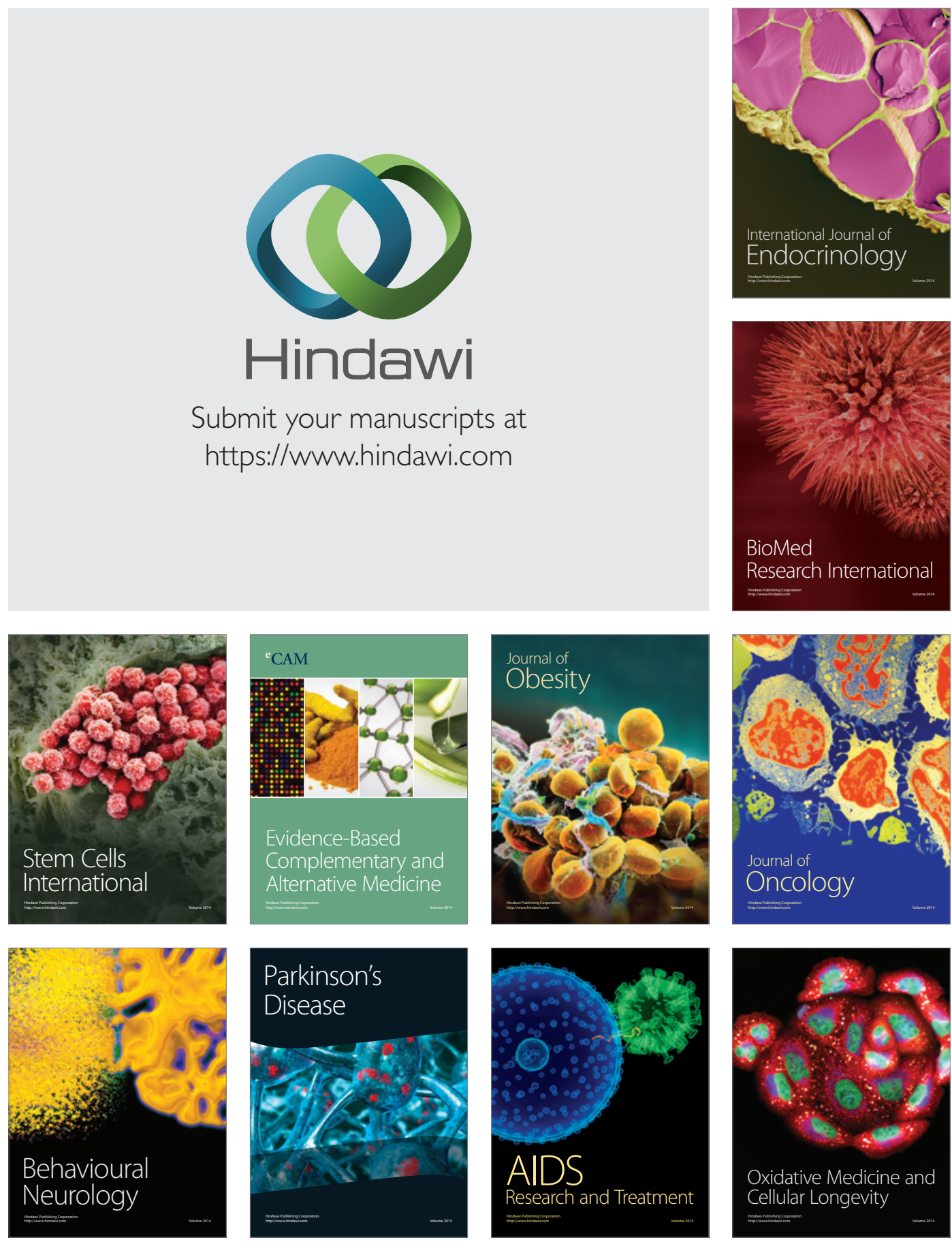